\title{
Multiset concepts in two-universe approximation spaces
}

\author{
O. A. Embaby ${ }^{1 *}$ (D) and Nadya A. Toumi ${ }^{2}$
}

\author{
*Correspondence: embabyosama@ \\ yahoo.com; embaby@science.tanta. \\ edu.eg \\ 'Department of Mathematics, \\ Faculty of Science, Tanta University, \\ Tanta, Egypt \\ Full list of author information is \\ available at the end of the article
}

\begin{abstract}
Rough set theory over two universes is a generalization of rough set model to find accurate approximations for uncertain concepts in information systems in which uncertainty arises from existence of interrelations between the three basic sets: objects, attributes, and decisions.

In this work, multisets are approximated in a crisp two-universe approximation space using binary ordinary relation and multi relation. The concept of two universe approximation is applied for defining lower and upper approximations of multisets. Properties of these approximations are investigated, and the deviations between them and corresponding notions are obtained; some counter examples are given. The suggested notions can help in the modification of the decision-making for events in which objects have repetitions such as patients visiting a doctor more than one time; an example for this case is given.
\end{abstract}

Keywords: Rough set, Multiset, Two universes approximation space

Mathematics Subject Classification: 54A05, 03E20, 68 U35

\section{Introduction}

A multiset is an unordered collection of objects in which, unlike the standard Cantorian set, the object is allowed to repeat. The word "multiset" often shortened to "mset" abbreviates the term "multiple membership set." In 1986, multiset theory was introduced by Yager [1]. Generalizations of the multiset concept were formalized by Blizard $[2,3]$. Applications of multisets to rough approximations were studied by Miyamoto [4]. Over the years, besides the sporadic evidence of the applications of multisets in logic, linguistics, and physics, a great number of them are witnessed in mathematics and computer science. An overview of the applications of multisets is presented by Singh et al. [5]. Algebraic structures for the multiset space were constructed by Ibrahim et al. [6]. Girish and John introduced multiset topologies induced by multiset relations and the continuity between multiset topological spaces [7, 8]. El-Sheikh et al. introduced separation axioms on multiset topological spaces and operators on multiset bitopological spaces $[9,10]$. The concepts of the exterior and boundary in the multiset topological space were introduced by Das and Mahanta [11]. Topological approximations of multisets are introduced by Abo-Tabl [12].

(c) The Author(s). 2020 Open Access This article is licensed under a Creative Commons Attribution 4.0 International License, which permits use, sharing, adaptation, distribution and reproduction in any medium or format, as long as you give appropriate credit to the original author(s) and the source, provide a link to the Creative Commons licence, and indicate if changes were made. The images or other third party material in this article are included in the article's Creative Commons licence, unless indicated otherwise in a credit line to the material. If material is not included in the article's Creative Commons licence and your intended use is not permitted by statutory regulation or exceeds the permitted use, you will need to obtain permission directly from the copyright holder. To view a copy of this licence, visit http://creativecommons.org/licenses/by/4.0/. 
The rough set theory was proposed by Pawlak $[13,14]$ for the study of intelligent systems characterized by insufficient and incomplete information. The rough set theory has been applied in artificial intelligence, medical diagnosis, pattern recognition, data mining, conflict analysis, and algebra [15-23]. Wong, Wang, and Yao generalized the rough set model using two distinct but related universes [24]. The formulation and interpretation of $U$ and $V$ and the compatibility relation between the two universes depend very much on the available knowledge and the domain of applications. For example, in a medical diagnosis system, $U$ can be a set of symptoms and $V$ a set of diseases. Thus, uncertainty arises when describing the interrelations between symptoms and diseases in clinical settings. In a specific group of patients, each patient may show many symptoms, just as each disease could have many symptoms.

Shen et al. [25] researched the variable precision rough set model over two universes. Yan et al. [26] studied the model of rough set over dual universe. Fuzzy rough set models over two universes were studied by Weihua et al. [27]. Many advances of the rough set model over two universes can be found in literature [28-33]. In 2019, Sun et al. [34] provided the theoretical model of multi granulation vague rough set over two universes. Another is to try making a new way to handle group decision-making problems under uncertainty based on multi granulation vague rough set theory and methodologies over two universes.

Grish et al. [35-37] applied multisets for constructing approximations for rough multisets in information multi systems, rough multisets, and its multiset topology and rough multiset relations.

The rest of the paper is organized as follows: In the "Preliminaries" section, basic concepts used in the work are presented. The purpose of the "Approximation of multisets in crisp approximation space" section is to study approximations of rough multiset in two-universe approximation space. While the "Approximation based on multi binary relation" section contains an application for using multi binary relation for rough set approximation.

\section{Preliminaries}

This section is devoted to present the basic concepts and properties of rough sets and multisets.

Definition 2.1 [37] An mset drawn from the set $A$ is represented by the count function $C_{M}$ defined as $C_{M}: A \rightarrow N$, where $N$ is the set of all non-negative integers. Here, $C_{M}(a)$ is the number of occurrences of the element $a$ in the mset $M$. The mset $M$ is drawn from set $A=\left\{a_{1}, a_{2}, \ldots, a_{n}\right\}$ and is written as $M=\left\{m_{1} / a_{1}, m_{2} / a_{2}, \ldots, m_{n} / a_{n}\right\}$, where $m_{i}$ is the number of occurrence of the element $a_{i}, i=1,2, \ldots, n$ in the mset $M$.

Definition 2.2 [37]. A domain $A$ is defined as a set of elements from which msets are constructed. The mset space $[A]^{\omega}$ is the class of all msets drawn from the set $A$ so that no element in the mset occurs more than $\omega$ times.

If $A=\left\{a_{1}, a_{2}, \ldots, a_{n}\right\}$, then $[A]^{\omega}=\left\{\left\{m_{1} / a_{1}, m_{2} / a_{2}, \ldots, m_{n} / a_{n}\right\}: m_{i} \in\{0,1,2, \ldots, \omega\}, i=1\right.$, $2, \ldots, n\}$.

The mset space $[A]^{\infty}$ is the class of all msets over a domain $A$ such that there is no limit in the number occurrences of an element in an mset.

Definition 2.3 [37] Let $M$ and $N$ be two msets drawn from a set $A$. Then:

1. $M=N$ if $C_{M}(a)=C_{N}(a) \quad \forall a \in A$

2. $M \subseteq N$ if $C_{M}(a) \leq C_{N}(a) \quad \forall a \in A$

3. $P=M \cup N$ if $C_{P}(a)=\max \left\{C_{M}(a), C_{N}(a)\right\} \quad \forall a \in A$ 
4. $P=M \cap N$ if $C_{P}(a)=\min \left\{C_{M}(a), C_{N}(a)\right\} \quad \forall a \in A$

5. $P=M \oplus N$ if $C_{P}(a)=\min \left\{C_{M}(a)+C_{N}(a), \omega\right\} \quad \forall a \in A$

6. $P=M \ominus N$ if $C_{P}(a)=\max \left\{C_{M}(a)-C_{N}(a), 0\right\} \quad \forall a \in A$, where $\oplus$ and $\ominus$ represent mset addition and subtraction, respectively.

Definition 2.4 [37] Let $M$ be an mset drawn from a set $A$. The support set of $M$ is a subset of $A$ defined by $M^{*}=\left\{a \in A: C_{M}(a)>0\right\}$, i.e., $M^{*}$ is an ordinary set and is also called the root set of $M$.

Definition 2.5 [37] Let $M$ be an mset drawn from a set $A$. If $C_{M}(a)=0 \forall a \in A$, then $M$ is called the empty mset and denoted by $\varnothing$.

Definition 2.6 [37] Let $M$ be an mset drawn from a set $A$ and $[A]^{\omega}$ be the mset space defined over $A$. Then, for any mset $M \in[A]^{\omega}$, the complement $M^{c}$ of $M$ in $[A]^{\omega}$ is an element of $[A]^{\omega}$ such that $C_{M^{c}}(a)=\omega-C_{M}(a) \forall a \in A$.

Definition 2.7 [37] The cardinality of an mset $M$ drawn from a set $A$ is defined byCard $M=\sum_{a \in A} C_{M}(a)$. It is also denoted by $|M|$.

Notation 2.1 [7] Let $M=\left\{m_{1} / x_{1}, m_{2} / x_{2}, \ldots, m_{n} / x_{n}\right\}$ be an mset drawn from the set $X=\left\{x_{1}, x_{2}, \ldots, x_{n}\right\}$ with $x$ appearing $m$ times in $M$. It is denoted by $x \in^{m} M$. The entry of the form $(m / x, n / y) / k$ denotes that $x$ is repeated $m$ times, $y$ is repeated $n$ times, and the pair $(x, y)$ is repeated $k$ times. The counts of the members of the domain and co-domain vary in relation to the counts of the $x$ coordinate and $y$ coordinate in $(m / x, n / y) / k$. For this purpose, let the notation $C_{1}(x, y)$ denotes the count of the first co-ordinate in the ordered pair $(x, y)$, and $C_{2}(x, y)$ denotes the count of the second co-ordinate in $(x, y)$.

Definition 2.8 [7] Let $M_{1}$ and $M_{2}$ be two msets drawn from a set $X$; then, the Cartesian product of $M_{1}$ and $M_{2}$ is defined by $M_{1} \times M_{2}=\left\{(m / x, n / y) / m n: x \in{ }^{m} M_{1}, \quad y \in{ }^{n} M_{2}\right\}$.

Definition 2.9 [7] A sub mset $R$ of $M \times M$ is said to be an mset relation on $M$ if every member $(m / x, n / y)$ of $R$ has a count, the product of $C_{1}(x, y)$ and $C_{2}(x, y)$. We denote $m /$ $x$ related to $n / y$ by $m / x R n / y$.

Definition 2.10 [38] Let $(U, V, R)$ be a two-universe approximation space. Then, the set-valued mappings $F$ and $G$ represent the successor and predecessor neighborhood operators, respectively, defined as follows:

$F: U \rightarrow P(V), F(a)=\{b \in V:(a, b) \in R\}, G: V \rightarrow P(U), G(b)=\{a \in U:(a, b) \in R\}$.

$F$ and $G$ can be naturally extended to a mapping from $P(U)$ to $P(V)$ (resp. $P(V)$ to $P(U))$ which are also denoted by $F$ and $G$ :

$F: P(U) \rightarrow P(V), F(A)=\cup\{F(a): a \in A\}, G: P(V) \rightarrow P(U), G(Y)=\cup\{G(b): b \in A\}$.

Lemma 2.1 [38] Let $(U, V, R)$ be a two-universe approximation space, if $R$ is a strong inverse serial relation, then for all $a_{1}, a_{2} \in U, F\left(A_{1}\right) \cap F\left(A_{2}\right) \neq \phi$ implies that $F\left(a_{1}\right)=F\left(a_{2}\right)$.

Proposition 2.1 [39] Let $R$ be an arbitrary binary relation on $U$. Then, $\forall A \in P(U)$ :

(i) $R$ is reflexive $\Leftrightarrow \underline{R}_{s}(A) \subseteq A \Leftrightarrow A \subseteq \bar{R}_{s}(A)$

(ii) $R$ is symmetric $\Leftrightarrow A \subseteq \underline{R}_{s}\left(\bar{R}_{s}(A)\right) \Leftrightarrow \bar{R}_{s}\left(\underline{R}_{s}(A)\right) \underline{\subseteq A}$

(iii) $R$ is transitive $\Leftrightarrow \underline{R}_{s}(A) \subseteq \underline{R}_{s}\left(\underline{R}_{s}(A)\right) \Leftrightarrow \bar{R}_{s}\left(\bar{R}_{s}(A)\right) \subseteq \bar{R}_{s}(A)$

(iv) $R$ is Euclidean $\Leftrightarrow \bar{R}_{s}(A) \subseteq \underline{R}_{s}\left(\bar{R}_{s}(A)\right) \Leftrightarrow \bar{R}_{s}\left(\underline{R}_{s}(A)\right) \subseteq \underline{R}_{s}(A)$

\section{Approximation of multisets in crisp approximation space}

Definition 3.1 Let $U$ and $V$ be two finite non-empty universes of discourse and $R \in$ $P(U \times V)$ be a binary relation from $U$ to $V$. The ordered triple $(U, V, R)$ is called a (twouniverse) approximation space. Let $B \in[V]^{w}$ be a multi set drawn from $V$. 
The lower and upper approximation of $B, \underline{R}_{s}(B)$ and $\bar{R}_{s}(B)$, with respect to the approximation space are multi set of $U$ whose membership functions, for each $a \in U$, are defined, respectively, by:

$$
\begin{aligned}
& C_{\underline{R}_{s}(B)}(a)=\min \left\{C_{B}(b): b \in F(a)\right\} \\
& C_{\bar{R}_{s}(B)}(a)=\max \left\{C_{B}(b): b \in F(a)\right\}
\end{aligned}
$$

where $F(a)$ is the successor neighborhood of $a$.

The ordered set pair $\left(\underline{R}_{s}(B), \bar{R}_{s}(B)\right)$ is referred to as a generalized rough multiset with respect to successor neighborhood, and $\underline{R}_{s}: P(V) \rightarrow P(U)$ and $\bar{R}_{s}: P(V) \rightarrow P(U)$ are referred to as lower and upper generalized rough multi approximation operators, respectively.

Definition 3.2 Let $(U, V, R)$ be a two-universe approximation space. Then, the lower and upper approximations of $A \in[U]^{w}$ are defined, respectively, as follows:

$$
\begin{aligned}
& C_{\underline{R}_{P}(A)}(b)=\min \left\{C_{A}(a): a \in G(b)\right\} \\
& C_{\bar{R}_{P}(A)}(b)=\max \left\{C_{A}(a): a \in G(b)\right\}
\end{aligned}
$$

where $G(b)$ is the predecessor neighborhood of $b$.

The pair $\left(\underline{R}_{P}(A), \bar{R}_{P}(A)\right)$ is referred to as a generalized rough multiset with respect to the predecessor neighborhood, and $\underline{R}_{P}: P(U) \rightarrow P(V)$ and $\bar{R}_{P}: P(U) \rightarrow P(V)$ are referred to as lower and upper rough multi approximation operators, respectively. If $\underline{R}_{P}(A)=\bar{R}_{P}(A)$, then $A$ is called an exact multiset; otherwise, $A$ is a rough multiset.

Proposition 3.1 In a two-universe model $(U, V, R)$ with the binary relation $R$, the approximation operators $\underline{R}_{P}$ and $\bar{R}_{P}$ satisfy the following properties for all $A, A_{1}$, $A_{2} \in[U]^{w}:$
$\left(L_{1}\right) \underline{R}_{P}(A)=\left(\bar{R}_{P}\left(A^{c}\right)\right)^{c}$.
$\left(L_{2}\right) \underline{R}_{P}(U)=V$.
(L) $\underline{R}_{P}\left(A_{1} \cap A_{2}\right)=\underline{R}_{P}\left(A_{1}\right) \cap \underline{R}_{P}\left(A_{2}\right)$.
$\left(L_{5}\right) A_{1} \subseteq A_{2} \Longrightarrow \underline{R}_{P}\left(A_{1}\right) \subseteq \underline{R}_{P}\left(A_{2}\right)$.
$\left(L_{4}\right) \underline{R}_{P}\left(A_{1} \cup A_{2}\right) \supseteq \underline{R}_{P}\left(A_{1}\right) \cup \underline{R}_{P}\left(A_{2}\right)$.
$\left(U_{2}\right) \bar{R}_{P}(\phi)=\phi$.
$\left(U_{1}\right) \bar{R}_{P}(A)=\left(\underline{R}_{P}\left(A^{c}\right)\right)^{c}$.
(U $\left.U_{4}\right) \bar{R}_{P}\left(A_{1} \cap A_{2}\right) \subseteq \bar{R}_{P}\left(A_{1}\right) \cap \bar{R}_{P}\left(A_{2}\right)$.
$\left(U_{3}\right) \bar{R}_{P}\left(A_{1} \cup A_{2}\right)=\bar{R}_{P}\left(A_{1}\right) \cup \bar{R}_{P}\left(A_{2}\right)$.
(U) $A_{1} \subseteq A_{2} \Longrightarrow \bar{R}_{P}\left(A_{1}\right) \subseteq \bar{R}_{P}\left(A_{2}\right)$.

Proof By the duality of approximation operators, we only need to prove the properties $L_{1}-L_{5}$.

$\left(L_{1}\right)$ For all $b \in V$, according to Definition 3.2, we can obtain:

$$
\begin{aligned}
\left.C_{\left[\bar{R}_{P}\left(A^{c}\right)\right.}\right]^{c}(b) & =w-\left\{\max \left\{C_{A^{c}}(a): a \in G(b)\right\}\right\}=w-\left\{\max \left\{w-C_{A}(a): a \in G(b)\right\}\right\} \\
& =w-\left\{w-\min \left\{C_{A}(\mathrm{a}): a \in G(b)\right\}\right\}=w-w+\min \left\{C_{A}(a): a \in G(b)\right\} \\
& =\min \left\{C_{A}(a): a \in G(b)\right\}=C_{\underline{R}_{P}(A)}(b) .
\end{aligned}
$$

Therefore, $\underline{R}_{P}(A)=\left(\bar{R}_{P}\left(A^{c}\right)\right)^{c}$.

$\left(L_{2}\right)$ Since $C_{U}(a)=1 \forall a \in U$ and $G(b) \subseteq U$, the $\min \left\{C_{U}(a): a \in G(b)\right\}=1$. Thus, $C_{\underline{R}_{p}}(U)($ $b)=\min \left\{C_{U}(a): a \in G(b)\right\}=1$ for all $b \in V$. Therefore, $\underline{R}_{P}(U)=V$.

$\left(L_{3}\right)$ Since $\forall b \in V$, 


$$
\begin{aligned}
C_{\underline{\underline{R}}_{P}\left(A_{1} \cap A_{2}\right)}(b) & =\min \left\{C_{\left(A_{1} \cap A_{2}\right)}(a): a \in G(b)\right\}=\min \left\{\min \left\{C_{A_{1}}(a), C_{A_{2}}(a)\right\}: a \in G(b)\right\} \\
& =\min \left\{\min \left\{C_{A_{1}}(a): a \in G(b)\right\}, \min \left\{C_{A_{2}}(a): a \in G(b)\right\}\right\} \\
& =\min \left\{C_{\underline{\underline{R}}_{P}\left(A_{1}\right)}(b), C_{\underline{R}_{P}\left(A_{2}\right)}(b)\right\}=C_{\underline{R}_{P}\left(A_{1}\right) \cap \underline{\underline{R}}_{P}\left(A_{2}\right)}(b) .
\end{aligned}
$$

Therefore, $\underline{R}_{P}\left(A_{1} \cap A_{2}\right)=\underline{R}_{P}\left(A_{1}\right) \cap \underline{R}_{P}\left(A_{2}\right)$.

$\left(L_{4}\right)$ For all $b \in V$, we can have:

$$
\begin{aligned}
C_{\underline{R}_{P}\left(A_{1} \cup A_{2}\right)}(b) & =\min \left\{C_{\left(A_{1} \cup A_{2}\right)}(a): a \in G(b)\right\}=\min \left\{\max \left\{C_{A_{1}}(a), C_{A_{2}}(a)\right\}: a \in G(b)\right\} \\
& \geq \max \left\{\min \left\{C_{A_{1}}(a): a \in G(b)\right\}, \min \left\{C_{A_{2}}(a): a \in G(b)\right\}\right\} \\
& =\max \left\{C_{\underline{R}_{P}\left(A_{1}\right)}(b), C_{\underline{R}_{P}\left(A_{2}\right)}(b)\right\}=C_{\underline{R}_{P}\left(A_{1}\right) \cap \underline{R}_{P}\left(A_{2}\right)}(b) .
\end{aligned}
$$

Hence, $\underline{R}_{P}\left(A_{1} \cup A_{2}\right) \underline{\underline{R}}_{P}\left(A_{1}\right) \cup \underline{R}_{P}\left(A_{2}\right)$.

$\left(L_{5}\right)$ Since $A_{1} \subseteq A_{2}$, then $\forall a \in U, C_{A_{1}}(a) \leq C_{A_{2}}(a)$. Thus, $C_{\underline{R}_{P}\left(A_{1}\right)}(b)=\min \left\{C_{A_{1}}(a): a \in\right.$ $G(b)\} \leq \min \left\{C_{A_{2}}(a): a \in G(b)\right\}=C_{\underline{R}_{P}\left(A_{2}\right)}(b)$.

Therefore, $\underline{R}_{P}\left(A_{1}\right) \subseteq \underline{R}_{P}\left(A_{2}\right)$.

The next proposition gives us characterizations of the rough multi lower and rough multi upper approximation operators based on different types of relations.

Proposition 3.2. Let $R \in P(U \times V)$ be an arbitrary binary relation. Then, $\forall A \in[U]^{w}$ :

(i) $R$ is inverse serial $\Leftrightarrow\left(L_{6}\right) \underline{R}_{P}(\phi)=\phi \Leftrightarrow\left(U_{6}\right) \bar{R}_{P}(U)=V \Leftrightarrow(L U) \underline{R}_{P}(A) \subseteq \bar{R}_{P}(A)$.

If $U=V$, then:

(ii) $\mathrm{R}$ is reflexive $\Leftrightarrow\left(L_{7}\right) \underline{R}_{P}(A) \subseteq A \Leftrightarrow\left(U_{7}\right) A \subseteq \bar{R}_{P}(A)$

(iii) $R$ is symmetric $\Leftrightarrow\left(L_{8}\right) A \subseteq \underline{R}_{P}\left(\bar{R}_{P}(A)\right) \Leftrightarrow\left(U_{8}\right) \bar{R}_{P}\left(\underline{R}_{P}(A)\right) \subseteq A$

(iv) $R$ is transitive $\Leftrightarrow\left(L_{9}\right) \underline{R}_{P}(A) \subseteq \underline{R}_{P}\left(\underline{R}_{P}(A)\right) \Leftrightarrow\left(U_{9}\right) \bar{R}_{P}\left(\bar{R}_{P}(A)\right) \subseteq \bar{R}_{P}(A)$

(v) $R$ is left Euclidean $\Leftrightarrow\left(L_{10}\right) \bar{R}_{P}(A) \subseteq \underline{R}_{P}\left(\bar{R}_{P}(A)\right) \Leftrightarrow\left(U_{10}\right) \bar{R}_{P}\left(\underline{R}_{P}(A)\right) \subseteq \underline{R}_{P}(A)$

Proof (i) Supposing that $R$ is an inverse serial relation, then for any $b \in V$, we have $G(b) \neq \phi$. Thus, $C_{\underline{R}_{P}(\phi)}(b)=\min \left\{C_{\phi}(a): a \in G(b)\right\}=0 \forall b \in V$. Therefore, $\underline{R}_{P}(\phi)=\phi$.

Conversely, assuming that $\underline{R}_{P}(\phi)=\phi$,i.e., $C_{\underline{R}_{P}(\phi)}(b)=\min \left\{C_{\phi}(a): a \in G(b)\right\}=0 \forall b$ $\in V$. If there exists $b_{\circ} \in V$ such that $G\left(b_{\circ}\right)=\phi$ then $C_{\underline{R}_{p}(\phi)}\left(b_{\circ}\right)=\min \left\{C_{\phi}(a): a \in G\left(b_{\circ}\right)\right\}$ $=\min \{\quad\}=$ undefined which contradicts the assumption. Thus, $G(b) \neq \phi \forall b \in V$,i.e., $R$ is an inverse serial. We can prove that $R$ is an inverse serial if and only if $\left(U_{6}\right) \bar{R}_{P}(U)=V$ by the duality of approximation operators. For the third part, $R$ is inverse serial if and only if $(L U) \underline{R}_{P}(A) \subseteq \bar{R}_{P}(A)$, and the proof is obvious.

(ii) By the duality, it is only to prove that $R$ is reflexive if and only if $\left(L_{7}\right) \underline{R}_{P}(A) \subseteq A$. Since $R$ is reflexive, then $\forall b \in V, b \in G(b)$, i.e., $\min \left\{C_{A}(a): a \in G(b)\right\} \leq C_{A}(b)$ which implies that $\underline{R}_{P}(A) \subseteq A$.

Conversely, assuming $\underline{R}_{P}(A) \subseteq A$ for all multi subset $A$ of $U$. Because a crisp set is a special case of a multiset, then $\underline{R}_{P}(A) \subseteq A$ for all $A \subseteq U$ and by proposition $2.1, R$ is a reflexive relation.

(iii) Assuming that $R$ is symmetric, then for all $a \in G(b)$, we have $b \in G(a)$. So, $\max \{-$ $\left.\min \left\{C_{A}(c): c \in G(a)\right\}: a \in G(b)\right\} \leq C_{A}(b)$.

Therefore, $\underline{R}_{P}\left(\bar{R}_{P}(A)\right) \subseteq A$. 
Conversely, assuming $\bar{R}_{P}\left(\underline{R}_{P}(A)\right) \subseteq A$ for all multi subset $A$ of $U$. Because a crisp set is a special case of a multiset, then $\bar{R}_{P}\left(\underline{R}_{P}(A)\right) \subseteq A$ for all $A \subseteq U$ and by proposition 2.1, $R$ is a symmetric relation. For the other statement, the proof is similar.

(iv) Supposing that $R$ is a transitive relation, then for all $a \in G(b)$, we have $G(a) \subseteq$ $G(b)$. Thus, $C_{\underline{R}_{p}\left(\underline{R}_{p}(A)\right)}(b)=\min \left\{\min \left\{C_{A}(a): c \in G(a)\right\}: a \in G(b)\right\} \geq \min \left\{\min \left\{C_{A}(c)\right.\right.$ $: c \in G(b)\}: a \in G(b)\}=\min \left\{C_{A}(c): c \in G(b)\right\}=\underline{R}_{\mathcal{P}}(A)(b)$.

Therefore, $\underline{R}_{P}(A) \subseteq \underline{R}_{P}\left(\underline{R}_{P}(A)\right)$.

The proof of the other side is similar to (iii).

(v) Assuming that $R$ is a left Euclidean relation, then for all $a \in G(b)$, we have $G(b) \subseteq$ $G(a)$. So, $\quad C_{\bar{R}_{P}\left(\underline{R}_{P}(A)\right)}(b)=\max \left\{\min \left\{C_{A}(c): c \in G(a)\right\}: a \in G(b)\right\} \leq \max \left\{\min \left\{C_{A}(c)\right.\right.$ $: c \in G(b)\}: a \in G(b)\}=\min \left\{C_{A}(c): c \in G(b)\right\}=C_{\underline{R}_{P}(A)}(b)$.

Therefore, $\bar{R}_{P}\left(\underline{R}_{P}(A)\right) \subseteq \underline{R}_{P}(A)$.

The proof of the other side is like (iii).

Remark 3.1 If $R \in P(U \times V)$ is a serial relation in a two-universe approximation space $(U, V, R)$, then the properties $L_{6}, U_{6}$, and $L U$ are not true in general, as shown in the following example:

Example 3.1 Let $U=\left\{a_{1}, a_{2}, a_{3}, a_{4}\right\}, V=\left\{b_{1}, b_{2}, b_{3}, b_{4}, b_{5}\right\}$, and $R$ be a binary relation from $U$ to $V$ defined as:

$$
\mathrm{R}=\left\{\left(a_{1}, b_{2}\right),\left(a_{1}, b_{4}\right),\left(a_{2}, b_{3}\right),\left(a_{2}, b_{4}\right),\left(a_{3}, b_{3}\right),\left(a_{4}, b_{1}\right),\left(a_{4}, b_{2}\right)\right\} .
$$

If $A \in[U]^{w}$ is a multiset drawn from $U$. Let $A=\left\{2 / a_{1}, 3 / a_{2}, 4 / a_{4}\right\}$.

Then, we have:

\begin{tabular}{llllll}
\hline & $b_{1}$ & $b_{2}$ & $b_{3}$ & $b_{4}$ & $b_{5}$ \\
\hline$C_{\underline{R}_{P}(A)}(b)$ & 4 & 2 & 0 & 2 & undefined \\
$C_{\bar{R}_{P(A)}}(b)$ & 4 & 4 & 3 & 3 & undefined \\
$C_{\underline{R}_{P}(\varphi)}(b)$ & 0 & 0 & 0 & 0 & undefined \\
$C_{\bar{R}_{p(U)}}(b)$ & 1 & 1 & 1 & 1 & undefined \\
\hline
\end{tabular}

Hence, $\underline{R}_{P}(\phi) \neq \phi, \bar{R}_{P}(U) \neq V$, and $\underline{R}_{P}(A) \neq \bar{R}_{P}(A)$, i.e., $L_{6}, U_{6}$, and $L U$ do not hold.

Remark 3.2 Let $R$ be any reflexive relation, then $\forall A \in[U]^{w}$ the properties $L_{8}-L_{10}$ and $U_{8}-U_{10}$ are not true in general. The following example shows this remark.

Example 3. 2 Let $U=\left\{a_{1}, a_{2}, a_{3}, a_{4}, a_{5}\right\}$ and $R$ be a reflexive relation on $U$ defined as $R=\left\{\left(a_{1}, a_{1}\right),\left(a_{1}, a_{2}\right),\left(a_{2}, a_{1}\right),\left(a_{2}, a_{2}\right),\left(a_{2}, a_{4}\right),\left(a_{3}, a_{3}\right),\left(a_{3}, a_{5}\right)\right.$,

$$
\left.\left(a_{4}, a_{2}\right),\left(a_{4}, a_{4}\right),\left(a_{5}, a_{2}\right),\left(a_{5}, a_{5}\right)\right\} \text {. }
$$

If $A$ and $B$ are multisets drawn from $U$ defined as $A=\left\{2 / a_{2}, 3 / a_{3}, 4 / a_{5}\right\}$ and $B=\left\{2 / a_{1}\right.$, $\left.3 / a_{2}, 1 / a_{4}, 4 / a_{5}\right\}$, then we have:

\begin{tabular}{llllll}
\hline & $a_{1}$ & $a_{2}$ & $a_{3}$ & $a_{4}$ & $a_{5}$ \\
\hline$C_{\underline{R}_{P}(B)}(a)$ & 2 & 1 & 0 & 1 & 0 \\
$C_{\underline{R}_{P}\left(\underline{R}_{p}(A)\right)}(a)$ & 1 & 0 & 0 & 1 & 0 \\
$C_{\bar{R}_{\rho}\left(\underline{R}_{P}(A)\right)}(a)$ & 0 & 3 & 3 & 0 & 3 \\
$C_{\bar{R}_{P}(A)}(a)$ & 2 & 4 & 3 & 2 & 4
\end{tabular}


Approximation of multisets in crisp approximation space (Continued)

\begin{tabular}{llllll}
\hline & $a_{1}$ & $a_{2}$ & $a_{3}$ & $a_{4}$ & $a_{5}$ \\
\hline$C_{\bar{R}_{P}\left(\bar{R}_{P}(B)\right)}(a)$ & 4 & 4 & 0 & 4 & 4 \\
$C_{\bar{R}_{P}(B)}(a)$ & 3 & 4 & 0 & 3 & 4 \\
$C_{\underline{R}_{P}\left(\bar{R}_{P}(A)\right)}(a)$ & 2 & 2 & 3 & 2 & 3 \\
\hline
\end{tabular}

Hence, $A \nsubseteq \underline{R}_{P}\left(\bar{R}_{P}(A)\right), \underline{R}_{P}(A) \nsubseteq \underline{R}_{P}\left(\underline{R}_{P}(A)\right), \bar{R}_{P}(A) \nsubseteq \underline{R}_{P}\left(\bar{R}_{P}(A)\right), \bar{R}_{P}\left(\underline{R}_{P}(A)\right) \nsubseteq A, \bar{R}_{P}\left(\bar{R}_{P}(A)\right)$ $\notin \bar{R}_{P}(A), \bar{R}_{P}\left(\underline{R}_{P}(A)\right) \notin \underline{R}_{P}(A)$,i.e., $L_{8}-L_{10}, U_{8}-U_{10}$ do not hold.

Remark 3.3 Let $R$ be any symmetric relation, then $\forall A \in[U]^{w}$ the properties $L_{6}, L_{7}$, $L_{9}, L_{10}, U_{6}, U_{7}, U_{9}, U_{10}$ and $L U$ are not true in general. The following example shows this remark.

Example 3.3 Let $U=\left\{a_{1}, a_{2}, a_{3}, a_{4}, a_{5}\right\}$ and $R$ be a symmetric relation on $U$ defined as $R=\left\{\left(a_{1}, a_{1}\right),\left(a_{1}, a_{2}\right),\left(a_{2}, a_{1}\right),\left(a_{2}, a_{4}\right),\left(a_{4}, a_{2}\right),\left(a_{4}, a_{4}\right),\left(a_{5}, a_{5}\right)\right\}$.

If $A$ is a multiset drawn from $U$ defined as $A=\left\{4 / a_{1}, 2 / a_{2}, 3 / a_{4}, 1 / a_{5}\right\}$, then we have:

\begin{tabular}{llllll}
\hline & $a_{1}$ & $a_{2}$ & $a_{3}$ & $a_{4}$ & $a_{5}$ \\
\hline$\underline{R}_{\mathcal{P}}(A)(a)$ & 2 & 3 & undefined & 2 & 1 \\
$\underline{R}_{\mathcal{P}}\left(\underline{R}_{\mathcal{P}}(A)\right)(a)$ & 2 & 2 & undefined & 2 & 1 \\
$\bar{R}_{\mathcal{P}}\left(\underline{R}_{\mathcal{P}}(A)\right)(a)$ & 3 & $\mathbf{2}$ & undefined & 3 & 1 \\
$\bar{R}_{\mathcal{P}}(A)(a)$ & 4 & 4 & undefined & 3 & 1 \\
$\bar{R}_{\mathcal{P}}\left(\bar{R}_{\mathcal{P}}(A)\right)(a)$ & 4 & 4 & undefined & 4 & 1 \\
$\underline{R}_{\mathcal{P}}\left(\bar{R}_{\mathcal{P}}(A)\right)(a)$ & 4 & 3 & undefined & 3 & 1 \\
$\underline{R}_{\mathcal{P}}(\varphi)(a)$ & 0 & 0 & undefined & 0 & 0 \\
$\bar{R}_{\mathcal{P}}(U)(a)$ & 1 & 1 & undefined & 1 & 1 \\
\hline
\end{tabular}

Hence, $\underline{R}_{P}(\phi) \neq \phi, \underline{R}_{P}(A) \nsubseteq A, \underline{R}_{P}(A) \nsubseteq \underline{R}_{P}\left(\underline{R}_{P}(A)\right), \bar{R}_{P}(A) \nsubseteq \underline{R}_{P}\left(\bar{R}_{P}(A)\right), \bar{R}_{P}(U) \neq U, A \nsubseteq \bar{R}_{P}(A$ ), $\bar{R}_{P}\left(\bar{R}_{P}(A)\right) \nsubseteq \bar{R}_{P}(A), \bar{R}_{P}\left(\underline{R}_{P}(A)\right) \nsubseteq \underline{R}_{P}(A)$ and $\underline{R}_{P}(A) \nsubseteq \bar{R}_{P}(A)$, i.e., $L_{6}, L_{7}, L_{9}, L_{10}$ and $U_{6}$, $U_{7}, U_{9}, U_{10}$ and $L U$ do not hold.

Remark 3.4 Let $R$ be any transitive relation, then $\forall A \in[U]^{w}$ the properties $L_{6}, L_{7}, L_{8}$, $L_{10}, U_{6}, U_{7}, U_{8}, U_{10}$ and $L U$ do not hold in general. The following example shows this remark.

Example 3.4 Let $U=\left\{a_{1}, a_{2}, a_{3}, a_{4}, a_{5}\right\}$ and $R$ be a transitive relation on $U$ defined as $R=\left\{\left(a_{1}, a_{2}\right),\left(a_{1}, a_{3}\right),\left(a_{2}, a_{3}\right),\left(a_{4}, a_{4}\right),\left(a_{5}, a_{2}\right),\left(a_{5}, a_{3}\right)\right\}$.

If $A$ is a multiset drawn from $U$ defined as $A=\left\{3 / a_{1}, 4 / a_{3}, 2 / a_{5}\right\}$ and $B=\left\{3 / a_{1}, 1 / a_{2}, 2 /\right.$ $\left.a_{4}, 4 / a_{5}\right\}$, then we have:

\begin{tabular}{llllll}
\hline & $a_{1}$ & $a_{2}$ & $a_{3}$ & $a_{4}$ & $a_{5}$ \\
\hline$\underline{R}_{\mathcal{P}}(A)(a)$ & undefined & 2 & 0 & 0 & undefined \\
$\bar{R}_{\mathcal{P}}\left(\underline{R}_{\mathcal{P}}(B)\right)(a)$ & undefined & 0 & 3 & 2 & undefined \\
$\bar{R}_{\mathcal{P}}(A)(a)$ & undefined & 3 & 3 & 0 & undefined \\
$\underline{R}_{\mathcal{P}}(B)(a)$ & undefined & 3 & 1 & 2 & undefined \\
$\underline{R}_{\mathcal{P}}\left(\bar{R}_{\mathcal{P}}(A)\right)(a)$ & undefined & 0 & 0 & 0 & undefined \\
$\underline{R}_{\mathcal{P}}(\varphi)(a)$ & undefined & 0 & 0 & 0 & undefined \\
$\bar{R}_{\mathcal{P}}(U)(a)$ & undefined & 1 & 1 & 1 & undefined \\
\hline
\end{tabular}


Hence, $\underline{R}_{P}(\phi) \neq \phi, \underline{R}_{P}(A) \nsubseteq A, A \nsubseteq \underline{R}_{P}\left(\bar{R}_{P}(A)\right), \bar{R}_{P}(A) \nsubseteq \underline{R}_{P}\left(\bar{R}_{P}(A)\right), \bar{R}_{P}(U) \neq V, A \nsubseteq \bar{R}_{P}(A), \bar{R}_{P}$ $\left(\underline{R}_{P}(A)\right) \nsubseteq A, \bar{R}_{P}\left(\underline{R}_{P}(A)\right) \nsubseteq \underline{R}_{P}(A)$ and $\underline{R}_{P}(A) \nsubseteq \bar{R}_{P}(A)$, i.e., $L_{6}, L_{7}, L_{8}, L_{10}, U_{6}, U_{7}, U_{8}, U_{10}$ and $L U$ do not hold.

Definition 3.4 A multi constant $\hat{\alpha}$ is a multiset in $U$ defined as:

$C_{\hat{\alpha}}(a)=\alpha \forall a \in U, \alpha \in N$.

Proposition 3.3 Let $(U, V, R)$ be a two- universe approximation space, the rough multi lower and upper approximation operators have the following properties for all $A_{j} \in[U]^{w}, j \in J$ which is an finite index set and for all $\alpha \in\{1,2,3, \ldots\}$,

$$
\begin{aligned}
& \text { (i) } \underline{R}_{P}\left(\cap_{j \in J} A_{j}\right)=\cap_{j \in J} \underline{R}_{P}\left(A_{j}\right) . \\
& \text { (ii) } \underline{R}_{P}\left(\cup_{j \in J} A_{\mathrm{j}}\right) \supseteq \cup_{j \in J} \underline{R}_{P}\left(A_{j}\right) . \\
& \text { (iii) } \underline{R}_{P}(A \cup \hat{\alpha})=\underline{R}_{P}(A) \cup \hat{\alpha} . \\
& \text { (iv) } \bar{R}_{P}\left(\cup_{j \in J} A_{j}\right)=\cup_{j \in J} \bar{R}_{P}\left(A_{j}\right) . \\
& \text { (v) } \overline{\mathrm{R}}_{P}\left(\cap_{\mathrm{j} \in J} \mathrm{~A}_{\mathrm{j}}\right) \subseteq \cap_{j \in J} \bar{R}_{P}\left(A_{j}\right) .
\end{aligned}
$$

Proof By the duality of approximation operators, we only need to prove the properties (i) - (iii).

(i) For each $b \in V$, we have:

$$
\begin{aligned}
& C_{\underline{R}_{P}\left(\cap_{j \in J} A_{j}\right)}(b)=\min \left\{C_{\left(\cap_{j \in J} A_{j}\right)}(a):(a) \in G(b)\right\} \\
& =\min \left\{\min \left\{C_{A_{j}}(a): j \in J\right\}: a \in G(b)\right\} \\
& =\min \left\{\min \left\{C_{A_{j}}(a): a \in G(b)\right\}: j \in J\right\}=\min \left\{C_{\underline{R}_{P}\left(A_{j}\right)}(b): j \in J\right\} \\
& =C_{n_{j \in J} \underline{R}_{P}\left(A_{j}\right)}(b) .
\end{aligned}
$$

(ii) Since $\forall(b) \in V$,

$$
\begin{aligned}
& C_{\underline{R}_{P}\left(\cup_{j \in J} A_{j}\right)}(b)=\min \left\{C_{\left(\cup_{j \in J} A_{j}\right)}(a):(a) \in G(b)\right\} \\
& =\min \left\{\max \left\{C_{A_{j}}(a): j \in J\right\}:(a) \in G(b)\right\} \\
& \geq \min \left\{C_{B_{j}}(c):(c) \in G(b)\right\}, \forall j \in J=C_{\underline{R}_{P}\left(A_{j}\right)}(b), \forall j \in J .
\end{aligned}
$$

Therefore, $C_{\underline{R}_{P}\left(\cup_{j \in J} A_{j}\right)}(b) \geq \max \left\{\underline{R}_{P}\left(A_{j}\right)(b), \forall j \in J\right\}=C_{\cup_{j \in J} \underline{R}_{P}\left(A_{j}\right)}(b)$.

(iii) For each $(b) \in V$, we have:

$$
\begin{aligned}
C_{\underline{R}_{P}(A \cup \hat{\alpha})}(b) & =\min \left\{C_{(A \cup \hat{\alpha})}(a):(a) \in G(b)\right\}=\min \left\{\max \left\{C_{A}(a), C_{\hat{\alpha}}(a)\right\}:(a) \in G(b)\right\} \\
& =\max \left\{\min \left\{C_{A}(a): a \in G(b)\right\}, C_{\hat{\alpha}}(a)\right\}=C_{\left(\underline{R}_{P}(A) \cup \hat{\alpha}\right)}(b) .
\end{aligned}
$$

Proposition 3.4 Let $(U, V, R)$ be a two-universe approximation space. Then, the following are equivalent $\forall \alpha \in N$

(i) $R$ is an inverse serial relation,

(ii) $\underline{R}_{P}(\hat{\alpha})=\hat{\alpha}$, 
(iii) $\bar{R}_{P}(\hat{\alpha})=\hat{\alpha}$.

Proof (i) $\Longrightarrow$ (ii) Let $R$ be an inverse serial relation, then we have $\underline{R}_{P}(\hat{\alpha})=\underline{R}_{P}(\hat{\alpha} \cup \phi)$ $=\hat{\alpha} \cup \underline{R}_{P}(\phi)=\hat{\alpha} \cup \phi=\hat{\alpha}$.

(ii) $\Longrightarrow$ (iii) Coming from the duality of approximation operators.

(iii) $\Longrightarrow$ (i) Assuming $\bar{R}_{P}(\hat{\alpha})=\hat{\alpha}$, since $U$ is a special case of $\hat{\alpha}$ which is $\alpha=w$. Then by assumption, we have $\bar{R}_{P}(U)=V$, i.e., $R$ is an inverse serial relation.

In the next three propositions, the connections of the approximation operators in definitions 2.7, and 3.1 are made, and the conditions under which these approximation operators made the equivalent are obtained.

Proposition 3.5 Let $(U, V, R)$ be a two-universe approximation space, then the following holds for all $A \in[U]^{w}$ and $B \in[V]^{w}$ :

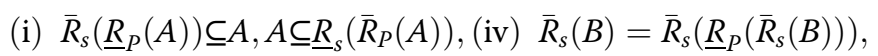

(ii) $\bar{R}_{P}\left(\underline{R}_{S}(B)\right) \subseteq B, B \subseteq \underline{R}_{P}\left(\bar{R}_{s}(B)\right),(\mathrm{v}) \underline{R}_{P}(A)=\underline{R}_{P}\left(\bar{R}_{S}\left(\underline{R}_{P}(A)\right)\right)$,

(iii) $\underline{R}_{s}(B)=\underline{R}_{s}\left(\bar{R}_{P}\left(\underline{R}_{s}(B)\right)\right),(\mathrm{vi}) \bar{R}_{P}(A)=\bar{R}_{P}\left(\underline{R}_{P}\left(\bar{R}_{P}(A)\right)\right)$

Proof (i) Since for every $a \in U$, we have either $F(a)=\phi$ or $F(a) \neq \phi$. If $F(a)=\phi$, then $C_{\bar{R}_{s}\left(\underline{R}_{P}(A)\right)}(a)=\max \left\{\min \left\{C_{A}(a): c \in G(b)\right\}: b \in F(a)\right\}=0$ and hence $\bar{R}_{s}\left(\underline{R}_{P}(A)\right) \subseteq A$. If $A(a) \neq \phi$, then we have $a \in G(b) \forall b \in A(a)$. Thus, $\max \left\{\min \left\{C_{A}(c): c \in G(b)\right\} b \in A(a)\right\} \leq$ $C_{A}(a)$, hence $\bar{R}_{S}\left(\underline{R}_{P}(A)\right) \subseteq A$. We can easily prove the other part by the duality of approximation operators.

(ii) is similar to (i).

(iii) - (vi) can be proved by the properties (i) and (ii).

Lemma 3.1 Let $(U, V, R)$ be a two-universe approximation space, $b \in V$; if $R$ is a strong inverse serial relation, then for all $a_{1}, a_{2} \in G(b)$,

$$
C_{\underline{R}_{s}(B)}\left(a_{1}\right)=C_{\underline{R}_{s}(B)}\left(a_{2}\right) ; C_{\bar{R}_{s}(B)}\left(a_{1}\right)=C_{\bar{R}_{s}(B)}\left(a_{2}\right) .
$$

Proof The proofs come directly from Lemma 2.1.

Proposition 3.6 Let $(U, V, R)$ be a two-universe approximation space with a strong inverse serial relation, then the following holds for all $A \in[U]^{w}$ and $B \in[V]^{w}$ :

(i) $\bar{R}_{P}\left(\underline{R}_{P}(B)\right)=\underline{R}_{P}\left(\bar{R}_{P}(B)\right)$

(ii) $\underline{R}_{P}\left(\bar{R}_{P}(B)\right)=\bar{R}_{P}\left(\bar{R}_{P}(B)\right)$.

Proof The proofs follow immediately from Lemma 3.1.

Proposition 3.7 Two pairs of lower approximation and upper approximation operators in definitions 2.7 and 3.2 are equivalent if and only if $R$ is a symmetric relation.

Proof Let $R$ be a symmetric relation on $U, A \in[U]^{w}$. Then for all $a \in U$, we have $F(a)=G(a)$, i.e.,

$$
\begin{aligned}
& C_{\underline{R}_{s}(A)}(a)=\min \left\{C_{A}(b): b \in F(a)\right\} \\
& =\min \left\{C_{A}(b): b \in G(a)\right\}=C_{\underline{R}_{P}(A)}(a) .
\end{aligned}
$$

Conversely, assuming $\underline{R}_{s}(A)=\underline{R}_{P}(A)$, since by the proposition 3.4, we have $\bar{R}_{P}\left(\underline{R}_{P}(A)\right.$ $\subseteq A$, by proposition 3.1 , and $R$ is a symmetric relation.

Proposition 3.8 Let $G=(U, R)$ be a generalized approximation space and $A$ be a multisubset of $U$. Then, the following holds: 
(i) If $R$ is symmetric then:

$$
\underline{R}_{P}(A)=\underline{R}_{P}\left(\bar{R}_{P}\left(\underline{R}_{P}(A)\right)\right) ; \bar{R}_{P}(A)=\bar{R}_{P}\left(\underline{R}_{P}\left(\bar{R}_{P}(A)\right)\right) .
$$

(ii) If $R$ is inverse serial and transitive then:

$$
\underline{R}_{P}(A) \subseteq \underline{R}_{P}\left(\bar{R}_{P}\left(\underline{R}_{P}(A)\right)\right) ; \bar{R}_{P}(A) \supseteq \bar{R}_{P}\left(\underline{R}_{P}\left(\bar{R}_{P}(A)\right)\right) .
$$

\section{Proof Obvious}

Example 3.5 Let $U=\left\{a_{1}, a_{2}, a_{3}, a_{4}\right\}$ a set of four patients and $V=\left\{\right.$ Fever $\left(b_{1}\right)$, Headache $\left(b_{2}\right)$, Stomachache $\left(b_{3}\right)$, Cough $\left(b_{4}\right)$, Myalgia $\left.\left(b_{5}\right)\right\}$ be five symptoms, if $R=\left\{\left(a_{1}\right.\right.$, $\left.\left.b_{2}\right),\left(a_{1}, b_{4}\right),\left(a_{2}, b_{3}\right),\left(a_{2}, b_{4}\right),\left(a_{3}, b_{3}\right),\left(a_{3}, b_{5}\right),\left(a_{4}, b_{1}\right),\left(a_{4}, b_{2}\right),\left(a_{4}, b_{5}\right)\right\}$

is a relation relating patients to symptoms. Let $A=\left\{3 / a_{1}, 0 / a_{2}, 3 / a_{3}, 5 / a_{4}\right\}$ represents a multiset of patients and times of visiting the doctor. Thus, using definition 2.10 , we have:

$$
G\left(b_{1}\right)=\left\{a_{4}\right\}, G\left(b_{2}\right)=\left\{a_{1}, a_{4}\right\}, G\left(b_{3}\right)=\left\{a_{2}, a_{3}\right\}, G\left(b_{4}\right)=\left\{a_{1}, a_{2}\right\}, G\left(b_{5}\right)=\left\{a_{3}, a_{4}\right\}
$$

and so, we get:

$$
\underline{R}_{P}(A)=\left\{5 / b_{1}, 3 / b_{2},{ }^{0} / b_{3}, 0 / b_{4},{ }^{2} / b_{5}\right\} \text { and } \bar{R}_{p}(A)=\left\{5 / b_{1}, 5 / b_{2},{ }^{2} / b_{3},{ }^{3} / b_{4},{ }^{5} / b_{5}\right\} .
$$

If $A=\left\{a_{1}, a_{3}, a_{4}\right\}$. By using the class $U / R^{-1}=\left\{\left\{a_{4}\right\},\left\{a_{1}, a_{4}\right\},\left\{a_{2}, a_{3}\right\},\left\{a_{1}, a_{2}\right\},\left\{a_{3}, a_{4}\right\}\right\}$, the lower and upper approximations using rough sets on one universe $U$ are $\underline{R}(A)=\{$ $\left.a_{1}, a_{3}, a_{4}\right\}=A$ and $\bar{R}(A)=\left\{a_{1}, a_{2}, a_{3}, a_{4}\right\}=U$. Clearly, this method does not have any deviations between the effectiveness of symptoms. But by using the multi approximations over the two universes $U$ and $V$, we have degree of effectiveness of $b_{1}$ which is $\frac{5}{5}, b_{2}$ which is $\frac{3}{5}, b_{3}$ which is $\frac{0}{2}, b_{4}$ which is $\frac{0}{3}$, and $b_{5}$ which is $\frac{2}{5}$.

\section{Approximation based on multi binary relation}

In this section, we aim to approximate rough sets in multi approximation spaces, study their properties, and provide a counter example.

Definition 4.1 Let $U$ and $V$ be two finite non-empty universes of discourse. Let Mand $N$ be two multisets drawn from $U$ and $V$, respectively. Let $R$ be a multi binary relation from $M$ to $N$. The ordered $(U, V, M, N, R)$ is called a two-universe multi approximation space. For any crisp set $A \subseteq U$, the lower and upper approximations of $A, \underline{R}(A)$ and $\bar{R}(A)$, with respect to the multi approximation space, are multisets drawn from $V$ whose count functions are defined respectively by:

For each $b \in V$,

$$
\begin{aligned}
& C_{\underline{R}(A)}(b)=\min \{m:(m / a) \in R(1 / b), a \in A\} \\
& C_{\bar{R}(A)}(b)=\max \{m:(m / a) \in R(1 / b), a \in A\}
\end{aligned}
$$

If for all $b \in V, C_{\underline{R}(A)}(b)=C_{\bar{R}(A)}$, then the set $A$ is definable (or exact) with respect to the multi approximation space $(U, V, M, N, R)$. Otherwise, the set $A$ is rough with respect to the multi approximation space.

Proposition 4.1 In a multi approximation space $(U, V,, M, N, R)$, the multi approximation operators satisfy the following properties for all $A, A_{1}, A_{2} \in P(U)$ : 
$\left(L_{3}^{\prime}\right) \underline{R}\left(A_{1} \cap A_{2}\right) \underline{\subseteq} \underline{R}\left(A_{1}\right) \cap \underline{R}\left(A_{2}\right) \quad\left(U_{3}\right) \quad \bar{R}\left(A_{1} \cup A_{2}\right)=\bar{R}\left(A_{1}\right) \cup \bar{R}\left(A_{2}\right)$

$\left(L_{4}^{\prime}\right) \underline{R}\left(A_{1} \cup A_{2}\right)=\underline{R}\left(A_{1}\right) \cup \underline{R}\left(A_{2}\right)\left(U_{4}\right) \bar{R}\left(A_{1} \cap A_{2}\right) \subseteq \bar{R}\left(A_{1}\right) \cap \bar{R}\left(A_{2}\right)$

$\left(L_{5}\right) \quad A_{1} \subseteq A_{2} \Longrightarrow \underline{R}\left(A_{1}\right) \subseteq \underline{R}\left(A_{2}\right)\left(U_{5}\right) \quad A_{1} \subseteq A_{2} \Longrightarrow \bar{R}\left(A_{1}\right) \subseteq \bar{R}\left(A_{2}\right)$.

$(L U) \quad \underline{R}(A) \subseteq \bar{R}(A)$.

Proof According to the duality of these properties, we only need to prove $\left(L^{\prime}{ }_{3}\right),\left(L^{\prime}{ }_{4}\right)$, $\left(L_{5}\right)$ and $(L U)$.

$\left(L_{3}\right)$ Since for all $b \in V$,

$$
\begin{aligned}
C_{\underline{R}\left(A_{1} \cap A_{2}\right)}(1 / b) & =\min \left\{m:(m / a) R(1 / b), a \in\left(A_{1} \cap A_{2}\right)\right\} \\
& \leq \min \left\{\min \left\{D:(m / a) \in R(1 / b), a \in A_{1}\right\}, \min \left\{m:(m / a) \in R(1 / b), a \in A_{2}\right\}\right\} \\
& \leq \min \left\{C_{\underline{R}\left(A_{1}\right)}(1 / b), C_{\underline{R}\left(A_{2}\right)}(1 / b)\right\} \subseteq \underline{R}\left(A_{1}\right) \cap \underline{R}\left(A_{2}\right) .
\end{aligned}
$$

Hence, $\underline{R}_{U}\left(A_{1} \cap A_{2}\right) \subseteq \underline{R}_{U}\left(A_{1}\right) \cap \underline{R}_{U}\left(A_{2}\right)$.

$\left(L_{4}\right)$ For all $b \in V$, we can have:

$$
\begin{aligned}
C_{\underline{R}\left(A_{1} \cup A_{2}\right)}(1 / b) & =\min \left\{m:(m / a) \in R(1 / b), a \in\left(A_{1} \cup A_{2}\right)\right\} \\
& =\min \left\{\max \left\{m:(m / a) \in R(1 / b), a \in\left(A_{1}\right), a \in\left(A_{2}\right)\right\}\right\} \\
& =\max \left\{\min \left\{m:(m / a) \in R(1 / b), a \in A_{1}\right\}, \min \left\{m:(m / a) \in(1 / b), a \in A_{2}\right\}\right\} \\
& =\max \left\{C_{\underline{R}\left(A_{1}\right)}(1 / b), C_{\underline{R}\left(A_{2}\right)}(1 / b)\right\}=C_{\underline{R}\left(A_{1}\right) \cup \underline{\underline{R}}\left(A_{2}\right)}(1 / b) .
\end{aligned}
$$

Hence, $\underline{R}\left(A_{1} \cup A_{2}\right)=\underline{R}\left(A_{1}\right) \cup \underline{R}\left(A_{2}\right)$.

$\left(L_{5}\right)$ Since $A_{1} \subseteq A_{2}$, then $\forall a \in U, A_{1} C_{A_{1}}(a) \leq C_{A_{2}}(a)$. Thus, $C_{\underline{R}\left(A_{1}\right)}(1 / b)=\min \{m:(m)$ a) $\left.\in R(1 / b), a \in A_{1}\right\} \leq \min \left\{m:(m / a) \in R(1 / b), a \in A_{2}\right\}=C_{\underline{R}\left(A_{2}\right)}(1 / b)$.

Therefore, $\underline{R}\left(A_{1}\right) \subseteq \underline{R}\left(A_{2}\right)$.

$(L U)$ For all $b \in V$, we can have:

$$
\begin{aligned}
& C_{\underline{R}(A)}(1 / b)=\min \{m:(m / a) \in R(1 / b), a \in A\} \\
& \leq \max \{m:(m / a) \in R(1 / b), a \in A\}=C_{\bar{R}(A)}(b) .
\end{aligned}
$$

Hence, $\underline{R}(A) \subseteq \bar{R}(A)$.

Remark 4.1 If $R \in[M \times N]^{w}$ is a multi binary relation in a two-universe approximation space $(U, V, M, N, R)$, then the following properties need not be true:

$$
\begin{aligned}
& \left(L_{1}\right) \underline{R}(A)=\left(\bar{R}\left(A^{c}\right)\right)^{c},\left(U_{1}\right) \bar{R}(A)=\left(\underline{R}\left(A^{c}\right)\right)^{c}, \\
& \left(L_{2}\right) \underline{R}(U)=V,\left(U_{2}\right) \bar{R}(\phi)=\phi, \\
& \left(L_{3}\right) \underline{R}\left(A_{1} \cap A_{2}\right)=\underline{R}\left(A_{1}\right) \cap \underline{R}\left(A_{2}\right),\left(U_{6}\right) \bar{R}(U)=V, \\
& \left(L_{4}\right) \underline{R}\left(A_{1} \cup A_{2}\right) \underline{\supseteq} \underline{R}\left(A_{1}\right) \cup \underline{R}\left(A_{2}\right) .
\end{aligned}
$$

The following example shows this remark:

Example 4.1 Let $U=\left\{a_{1}, a_{2}, a_{3}, a_{4}, a_{5}, a_{6}, a_{7}\right\}, V=\left\{b_{1}, b_{2}, b_{3}, b_{4}, b_{5}\right\}$. Let $M$ be a multiset drawn from $U$ and $N$ be a multiset drawn from $V$ shath that $M=\left\{1 / a_{1}, 2 / a_{2}, 2\right.$ / $\left.a_{3}, 1 / a_{4}, 3 / a_{5}, 2 / a_{6}, 4 / a_{7}\right\}$ and $N=\left\{2 / b_{1}, 3 / b_{3}, 1 / b_{4}, 4 / b_{5}, 3 / b_{6}\right\}$ and $R$ be a multi binary relation from $M$ to $N$ defined as: 


$$
\begin{aligned}
R=\{ & \left(1 / a_{1}, 2 / b_{1}\right) / 2,\left(1 / a_{1}, 3 / b_{3}\right) / 3,\left(1 / a_{1}, 1 / b_{4}\right) / 1,\left(2 / a_{2}, 3 / b_{3}\right) / 6,\left(2 / a_{2}, 1 / b_{4}\right) / 2 \\
& \left(2 / a_{2}, 4 / b_{5}\right) / 8,\left(2 / a_{3}, 2 / b_{1}\right) / 4,\left(2 / a_{3}, 4 / b_{5}\right) / 8,\left(2 / a_{3}, 3 / b_{6}\right) / 6,\left(1 / a_{4}, 3 / b_{3}\right) / 3 \\
& \left(1 / a_{4}, 1 / b_{4}\right) / 1,\left(3 / a_{5}, 2 / b_{1}\right) / 6,\left(3 / a_{5}, 3 / b_{3}\right) / 9,\left(3 / a_{5}, 1 / b_{4}\right) / 3,\left(3 / a_{5}, 4 / b_{5}\right) / 12, \\
& \left(2 / a_{6}, 2 / b_{1}\right) / 4,\left(2 / a_{6}, 3 / b_{3}\right) / 6,\left(2 / a_{6}, 1 / b_{4}\right) / 2,\left(4 / a_{7}, 2 / b_{1}\right) / 8,\left(4 / a_{7}, 1 / b_{4}\right) / 4 \\
& \left.\left(4 / a_{7} 4 / b_{5}\right) / 16\right\}
\end{aligned}
$$

If $A$ is subset of $U$, defined as $A=A_{1}=\left\{a_{1}, a_{3}, a_{4}, a_{7}\right\}$ and $A_{2}=\left\{a_{1}, a_{2}, a_{4}, a_{6}\right\}$, then we have:

\begin{tabular}{lllllll}
\hline & $b_{1}$ & $b_{2}$ & $b_{3}$ & $b_{4}$ & $b_{5}$ & $b_{6}$ \\
\hline$C_{\underline{R}\left(A_{1}\right)}(1 / b)$ & 1 & 0 & 1 & 1 & 2 & 2 \\
$C_{\bar{R}\left(A_{1}\right)}(1 / b)$ & 4 & 0 & 1 & 4 & 4 & 2 \\
$C_{\underline{R}\left(A_{2}\right)}(1 / b)$ & 1 & 0 & 1 & 1 & 2 & 0 \\
$C_{\bar{R}\left(A_{2}\right)}(1 / b)$ & 2 & 0 & 2 & 2 & 2 & 0 \\
$C_{\underline{R}\left(A_{1} \cap A_{2}\right)}(1 / b)$ & 1 & 0 & 1 & 1 & 0 & 0 \\
$C_{\underline{R}\left(A_{\cdot}\right)} \cap C_{\underline{R}\left(A_{2}\right)}(1 / b)$ & 1 & 0 & 1 & 1 & 2 & 0 \\
$C_{\underline{R}\left(A_{\cdot}\right)} \cup C_{\underline{R}\left(A_{2}\right)}(1 / b)$ & 1 & 0 & 1 & 1 & 2 & 2 \\
$C_{\underline{R}\left(A_{1} \cup A_{2}\right)}(1 / b)$ & 1 & 0 & 1 & 1 & 2 & 2 \\
$C_{\left(\underline{R}\left(A^{c}\right)\right)^{c}(1 / b)}$ & 5 & 6 & 5 & 5 & 4 & 4 \\
$C_{\left(\bar{R}\left(A^{c}\right)\right)^{c}(1 / b)}$ & 3 & 6 & 3 & 3 & 3 & 6 \\
$C_{\underline{R}(\varphi)}(1 / b)$ & 0 & undefined & 0 & 0 & 0 & 0 \\
$C_{\bar{R}(\varphi)}(1 / b)$ & 0 & undefined & 0 & 0 & 0 & 0 \\
$C_{\underline{R}(U)}(1 / b)$ & 1 & 0 & 1 & 1 & 2 & 0 \\
$C_{\bar{R}(U)}(1 / b)$ & 4 & 0 & 3 & 4 & 4 & 2 \\
\hline
\end{tabular}

\section{Conclusion and future work}

The multiset approximations suggested in this work can help to compute measures and ordering of effectiveness and certainty of concepts in information systems. More work on using multi relation to approximate rough multi sets will be discussed in the future. Also, the use of a relation between two universes (objects and attributes) can be extended to construct another relation between attributes and decisions for constructing a rough set model over three universes.

Acknowledgements

The authors would like to thank the referees for providing very helpful comments and suggestions that helped in improving the quality of the paper.

\section{Authors' contributions}

The first and second author participated equally in all stages of the manuscript. All authors read and approved the final manuscript.

Funding

Not applicable. 


\section{Competing interests}

The authors declare that they have no competing interests.

\section{Author details}

${ }^{1}$ Department of Mathematics, Faculty of Science, Tanta University, Tanta, Egypt. ${ }^{2}$ Department of Mathematics, Faculty of Science, The 7th of April University, Al- Zawia, Libya.

Received: 20 January 2020 Accepted: 28 July 2020

Published online: 02 October 2020

\section{References}

1. Yager, R.R.: On the theory of bags. Int General Syst. 13, 23-37 (1986)

2. Blizard, W.D.: Multiset theory. Notre Dame Journal of Formal Logic. 30(1), 36-66 (1989)

3. Blizard, W.D.: Real -valued multisets and fuzzy sets theory. Fuzzy Sets and Systems. 33, 77-97 (1989)

4. Miyamoto, S.: Generalizations of multisets and rough approximations. International Journal of Intelligent Systems. 19, 639-652 (2004)

5. Singh, D., Ibrahim, A.M., Yohanna, T., Singh, J.N.: An overview of the applications of multisets. Novi SAD J. MATH. 37(2), 73-92 (2007)

6. Ibrahim, M., Singh, D., Singh, J.N.: An outline of multiset space algebra. International Journal of Algebra. 5(31), 1515-1525 (2011)

7. Girish, K.P., John, S.J.: Relations and functions in multiset context. Information Sciences. 2, 37-52 (2009)

8. Girish, K.P., John, S.J.: Multiset Topologies induced by multiset relations. Information Sciences. 188, 298-313 (2012)

9. El-Sheikh, S., Omar, R., Raafat, M.: Separation axioms on multiset topological spaces. Journal of New Theory. 7, 11-12 (2015)

10. El-Sheikh, S., Omar, R., Raafat, M.: Operators on multiset bitopological spaces. South Asian Journal of Mathematics. 6(1), 1-9 (2016)

11. Das, D., Mahanta, J.: Boundary and exterior of a multiset topology, the journal of new theory. 12, 75-84 (2016).

12. Abo-Tabl, E.A.: Topological approximations of multisets. Journal of the Egyptian Mathematical Society. 21, 123-132 (2013)

13. Pawlak, Z:: Rough sets theoretical aspects of reasoning about data, Kluwer Academic Publishers, Dordrecht 9 (1991)

14. Pawlak, Z:: Rough sets. Int. J. of Information and Computer Sciences. 11(5), 341-356 (1982)

15. Cheng, W., Mo, Z.W., Wang, J.: Notes on the lower and upper approximations in fuzzy group and rough ideals in semigroups. Inf. Sci. 177, 5134-5140 (2007)

16. Dempster, P.: Upper and lower probabilities induced by a multivalued mapping. Ann Math stat. 38, 325-339 (1967)

17. Dntsch, G.: Gediga, Uncertainty measures of rough sets prediction. Artif. Intell. 106, 109-137 (1998)

18. Jensen, R., Shen, Q.: Fuzzy -rough sets assisted attribute selection. IEEE Trans Fuzzy Syst. 15, 73-89 (2007)

19. Mi, J.S., Zhang, W.X.: An axiomatic characterization of a fuzzy generalization of rough sets. Inf. Sci. 160, $235-249$ (2004)

20. Z. Pawlak Rough sets, decision algorithms and Bayes's theorem. Eur .J. Oper. Res. 136, 181-189 (2002)

21. Pawlak, Z., Skowron, A.: Rudiments of rough sets. Inf. Sci. 177, 3-27 (2007)

22. Pawlak, Z: Some remarks on conflict analysis,. Eur. J. Oper. Res. 166, 649-654 (2005)

23. Ananthanarayana, V.S., Narasimha, M.M., Subramanian, D.K.: Tree structure for efficient data mining using rough sets, Pattern Recognit Lett. 24 851-862 (2003)

24. Shen, Y., Wang, F.: Variable precision rough set model over two universes and its properties. Soft Computing. 15(3), 557-567 (2011)

25. Yan, R.X., Zheng, J.G., Liu, J.L., Zhai, Y.M.: Research on the model of rough set over dual universes. Knowl. Based Syst. 23, 817-822 (2010)

26. Weihua, X., Wenxin, S., Yufeng, L., Wenxiu, Z:: Fuzzy rough set models over two universes. Int. J. Mach. Learn. \& Cyber. 4, 631-645 (2013)

27. Li, T.J., Zhang, W.X.: Rough fuzzy approximations on two universes of discourse, Inf. Sci. 178, 892-906 (2008)

28. Liu, G.L.: Rough set theory based on two universal sets and its applications. Knowl. Based Syst. 23, 110-115 (2010)

29. Pei, D.W., Xu, Z.B.: Rough set models on two universes. Int. J. Gen. Syst. 33, 569-581 (2004)

30. Shu, L., He, X.Z:: Rough set model with double universe of discourse, Proceedings of the IEEE international conference on Information Reuse and Integration (IRI '07), Las Vegas, Nev, USA. August. 492-495 (2007)

31. Sun, B.Z., Ma, W.M.: Fuzzy rough set model on two different universes and its application. Appl. Math. Model. 35, 17981809 (2011)

32. Zhang, H.Y., Zhang, W.X., Wu, W.Z.: On characterization of the generalized interval-valued fuzzy rough sets on two universes of discourse. Int. J. Approx. Reason. 51(1), 56-70 (2009)

33. Sun, B., Ma, W., Chen, X., Zhang, X.: Multigranulation vague rough set over two universes and its application to group decisionmaking. Soft Computing. 23, 8927-8956 (2019)

34. Girish K.P., Sunil J.J.: Rough multisets and information multisystems, Advances in Decision sciences, Volume 2011, Article number 495392

35. Girish K.P., Sunil J.J.: Rough multisets and its multiset topology, J.F. Peters et al. (Eds.): Transactions on Rough Sets XIV, LNCS 6600, 62-80, 2011, Springer-Verlag, Berlin (2011)

36. Girish K.P., Sunil J.J.: On rough multiset relations, Int. J. of Granular Computing, Rough sets and Intelligent Systems. 3(4), 306-326 (2014)

37. Jena, S.P., Gosh, S.K., Tripathy, B.K.: On the theory of bags and lists. Information sciences. 132, 241-254 (2001)

38. Yao, Y.Y., Wong, S.K., Lin, T.Y.: A review of rough set models. In: Lin, T.Y., Cercone, N. (eds.) Rough sets and data mining: analysis for imprecise data, pp. 47-75. Kluwer Academic publishers, Boston (1997)

39. Yao, Y.Y.: Relational interpretations of neighborhood operators and rough set approximation operators. Information Sciences. 111, 239-259 (1998)

\section{Publisher's Note}

Springer Nature remains neutral with regard to jurisdictional claims in published maps and institutional affiliations. 